\title{
The Dynamic Envelope of a Fusion Type 2 Virus - Reorganizations Observed during Prefusion Stages in Semliki Forest Virus
}

\author{
S.-R. Wu, ${ }^{*}$ L. Haag, ${ }^{*}$ L. Hammar, ${ }^{*}$ B.-M. Wu, ${ }^{*}$ H. Garoff, ${ }^{*}$ L. Xing, ${ }^{* *}$ K. Murata*** and R.H. \\ Cheng $* * *$ \\ * Department of Biosciences and Nutrition, Karolinska Institutet, S 14157 Huddinge, SWEDEN. \\ ** Section of Molecular and Cellular Biology, University of California, Davis, CA 95616, USA \\ *** National Institute of Advanced Industrial Science and Technology, Tokyo, 100-8921, JAPAN
}

Receptor interaction and release of its genome into the target cell are fundamental functions executed by a virus particle. With enveloped viruses this includes a membrane fusion step. Two types of virus-induced fusion mechanisms observed. The Semliki Forest Virus, SFV, is the fusion type 2 virus, also prevailing in some other Toga and Flaviviruses. The characteristics for this mechanism are that the fusion loop is an integral sequence at the end of the elongated fusion protein. The SFV is activated for fusion by acidification, reflecting that it infects by fusion with the endosomal membrane. The SFV glycoprotein E2 and its envelope companion, the fusion protein E1, are both anchored in the viral membrane and form an external shell domain with protruding trimeric spikes. These are organized in a $\mathrm{T}=4$ icosahedral lattice pattern. In the sub-membrane domain there is a contact with the nucleocapsid protein that is arranged in a phase shifted $\mathrm{T}=4$ pattern. We have earlier shown that SFV surface domains rearrange during acidification in a sequential manner before the fusion loop is exposed for external interaction [1-2]. This occurs while expanding, but the particle morphology is essentially retained in shape, as shown in Fig. 1. Now we show that the shell and stalk of the spike domain start to deteriorate at a stage when the fusion loop is exposed and the association of the two proteins in the spike head still remains tight, as shown in Fig. 2. Homotrimers of the fusion protein E1 has been implied as a post fusion structure [3-4]. Considering the organization of the glycoproteins in the virus envelope, we anticipate an elaborate cooperation between the two proteins, beyond the control of fusion-loop-exposure and that drives the membrane merging stage of fusion. This implies a more prominent role for the E2 protein in guiding the membrane close encounter and merging than has been earlier anticipated.

\section{References}

[1] L. Haag, , H. Garoff, L. Xing, L. Hammar, S.T. Kan and R.H. Cheng (2002). Embo J 21(17): 4402-10.

[2] L. Hammar, S. Markarian, L. Haag, H. Lankinen, A. Salmi and R.H. Cheng (2003). J Biol Chem 278(9): 7189-98.

[3] D.L. Gibbons, M.C. Vaney, A. Roussel, A. Vigouroux, B. Reilly, J. Lepault, M. Kielian and F.A. Rey (2004). Nature 427: 320-325.

[4] A. Roussel , J. Lescar, M.C. Vaney, G. Wengler, G. Wengler and F.A. Rey (2006). Structure 14: 75-86.

[5] This work has been supported by the European framework program 6, the Swedish Medical Research Council, Knowledge Foundation through the Industrial PhD program in Medical Bioinformatics between the Karolinska Institutet and the Crystal Research Company. 


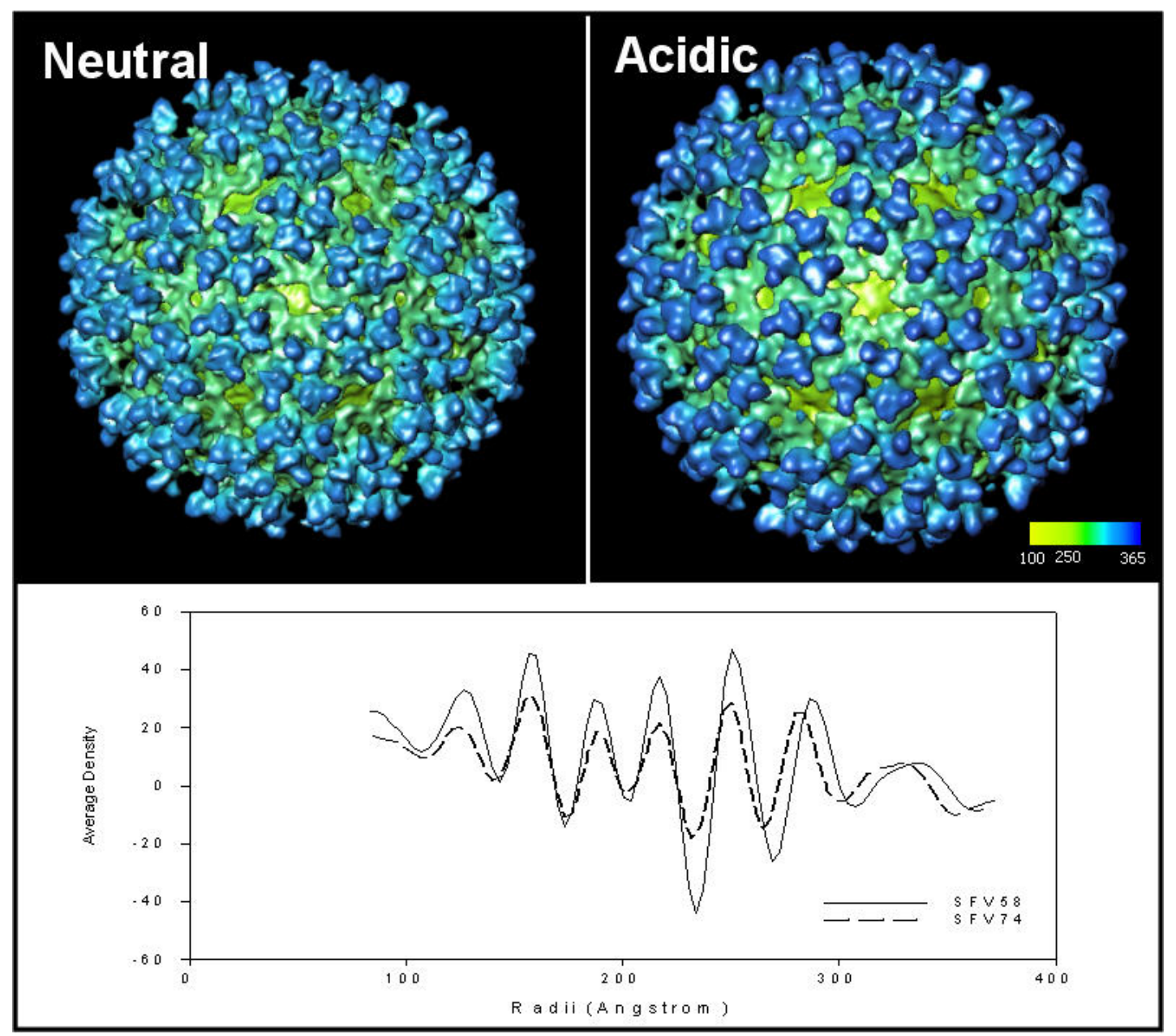

FIG. 1 Reconstructions derived from Cryo-EM image data of SFV particles treated at neutral (top, left) and acidic $\mathrm{pH}$ (top, right). The surface renderings, at 100\% mass, are viewed along the 2-fold axis and represent maps corrected to the same resolution of $\sim 15 \AA$. Bottom panel show the corresponding 1-D plots of average radial density.

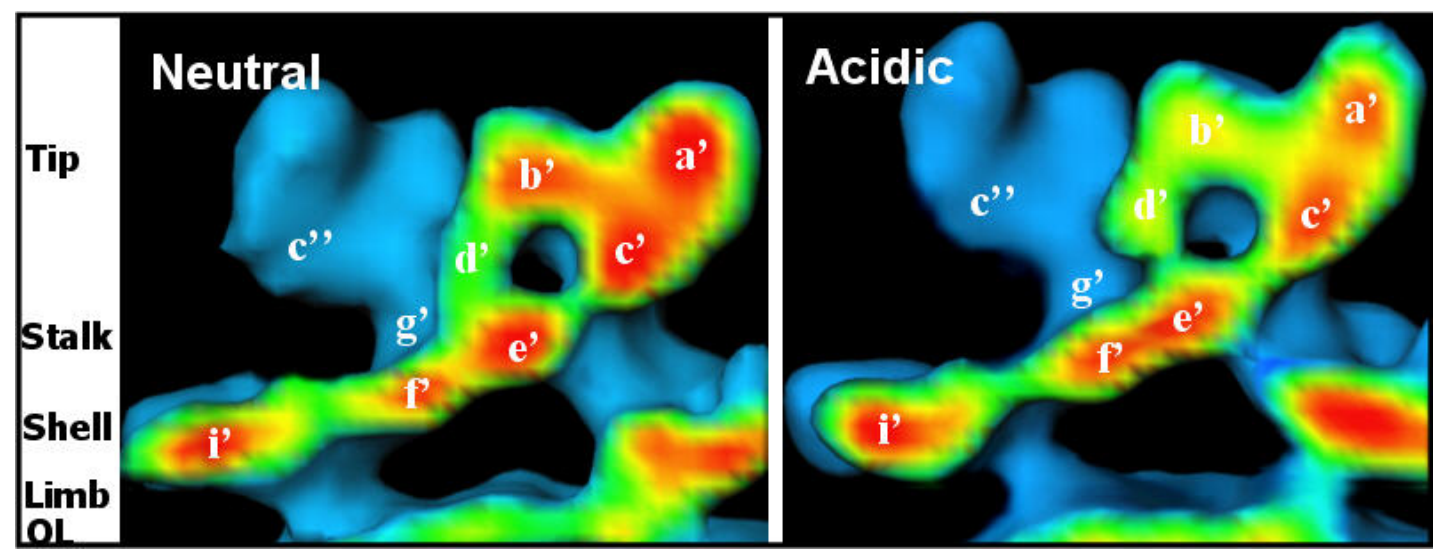

FIG. 2 Side views of sections through the head, stalk and shell domains of the 3-fold spike to demonstrate the $\mathrm{pH}$ dependent movement of major density nodes. The blue to red color-coding represents the gradient in gravity intensity. 